\title{
ESTADO BUCODENTAL DE ADULTOS MAYORES INSTITUCIONALIZADOS MEDIANTE UN PROGRAMA PÚBLICO EN VILLAVICENCIO, DURANTE EL PRIMER SEMESTRE DE 2014
}

Claudia Patricia Rodas Avellaneda ${ }^{1}$, María del Pilar Angarita Díaz ${ }^{2}$, Luis Francisco Nemocón Ramírez ${ }^{3}$, Luis Alexys Pinzón Castro ${ }^{4}$, Yenny Tatiana Robayo Herrera ${ }^{5}$, Inés Leonilde Rodríguez Baquero ${ }^{6}$, Rocío del Pilar González Sánchez ${ }^{7}$

\section{Resumen}

Introducción. El proceso de envejecimiento implica cambios fisiológicos que, junto con otros factores, pueden derivar en enfermedades que incluyen las de cavidad oral. La caries, la enfermedad periodontal y las lesiones de la mucosa son las más frecuentes y su presencia condiciona en gran medida la calidad de vida del adulto mayor.

Objetivo. Determinar el estado de salud bucodental de personas mayores institucionalizadas mediante un programa público de Villavicencio, en tres hogares geriátricos. Métodos. Se realizó un estudio descriptivo de adultos mayores donde se recolectó información personal (datos e historia clínica) y se aplicaron los índices (COP-D, Silness y Löe modificado, CPITN y GOHAI). Además, se recolectó información sobre manifestaciones en tejidos blandos y valoración del edentulismo. Resultados. El 48,5\% de la población era totalmente edéndula y el $51,5 \%$ aún conservaban dientes, pero con un promedio de pérdida de $18( \pm 7,2)$ dientes. De esta población, el $85,7 \%$ de los adultos mayores presentaron caries, el 74,1\% presentó un factor de riesgo alto en el índice de placa bacteriana y gingival, el 33,3\% presentaban enfermedad periodontal. Además, se detectó que el $19 \%$ de la población presentaba alguna lesión en la mucosa y que el $76,5 \%$ tenía una baja percepción de la calidad de vida relacionada con su salud bucal.

Conclusión: mediante la utilización de diferentes índices e instrumentos de valoración, se logró conocer el estado bucodental de adultos mayores institucionalizados bajo un programa público. Los resultados indican la necesidad de implementar estrategias y tratamientos que contribuyan a mejorar la salud oral de esta población vulnerable.

Palabras clave: anciano, higiene bucal, salud bucal.

Especialista en Gerencia en servicios de salud, odontóloga, profesora auxiliar. Programa de Odontología, Universidad Cooperativa de Colombia, sede Villavicencio (Meta). claudiap.rodas@campusucc.edu.co

${ }^{2}$ Ph.D. en Biotecnología, Ms en Cooperación al Desarrollo especialidad Planificación del Desarrollo Local, Microbiología Agrícola y Veterinaria. Profesora instructor. Programa de Odontología, Universidad Cooperativa de Colombia, sede Villavicencio (Meta), Colombia. maria.angaritad@campusucc.edu.co

${ }^{3}$ Especialista en odontología legal y forense. Especialista en implantología oral y reconstructiva. Profesor instructor. Programa de Odontología, Universidad Cooperativa de Colombia, sede Villavicencio (Meta), Colombia. Luis. Nemocon@campusucc.edu.co

${ }^{4}$ Ph.D. en Estadística y Matemáticas aplicadas. Ms. En Ciencias exactas y naturales, Lic. En Matemáticas. Profesor Instructor. Programa de Psicología. Universidad Cooperativa de Colombia. Sede Villavicencio. lapinzonc@unal. edu.co

${ }^{5}$ Especialista en Cirugía y Patología Oral. Odontóloga. Profesora instructora. Programa de Odontología, Universidad Cooperativa de Colombia, sede Villavicencio (Meta),Colombia. yenny.Robayo@campusucc.edu.co

${ }^{6}$ Especialista en Bioética. Odontóloga, profesora auxiliar Programa de Odontología, Universidad Cooperativa de Colombia, sede Villavicencio (Meta), Colombia. ines.rodriguezb@campusucc.edu.co

Especialista en Periodoncia. Odontóloga. Im_pily@yahoo.com 
ORAL HEALTH OF OLDER ADULTS INSTITUTIONALIZED AS PART OF A PUBLIC PROGRAM IN VILLAVICENCIO IN THE FIRST SEMESTER OF 2014

Claudia Patricia Rodas Avellaneda, María del Pilar Angarita Díaz, Luis Francisco Nemocón Ramírez, Luis Alexys Pinzón Castro, Yenny Tatiana Robayo Herrera, Inés Leonilde Rodríguez Baquero, Rocío del Pilar González Sánchez

\section{Abstract}

Introduction. The aging process implies physiological changes, which, along with other factors may lead to diseases including those of the oral cavity. Caries, periodontal disease, and mucosal lesions are among the most frequent and their presence affects to a great extent the quality of life of older adults.

Objective. To determine the oral health of older adults in three Social Protection Centers in Villavicencio. Method: A descriptive study of older adults in which Personal information (clinical data and history) was gathered and the following indices applied: COP-D, the Modified Silness and Löe, CPITN and GOHAI. It was also gathered information regarding manifestations on soft tissue and the assessment of edentulism. Results: $48.5 \%$ of the population was completely edentulous and $51.5 \%$ still had some teeth, but with an average tooth-loss rate of $18( \pm 7.2)$ teeth. Among this population, $85.7 \%$ had caries, $74.1 \%$ presented a high-risk factor on the Plaque Index (PI) and Gingival Index (GI), and 33.3\% presented periodontal disease. It was also detected that $19 \%$ of the population presented some form of mucosal lesion and that $76.5 \%$ had a low perception of their quality of life in relation to oral health.

Conclusion. Through the use of different indices and assessment instruments, it was possible elucidate facts regarding the oral health of older adults institutionalized as part of a public program in Villavicencio. The results highlight the need to implement strategies and treatments that contribute to improving the oral health of this vulnerable population. 


\section{SAÚDE BUCAL DE IDOSOS INSTITUCIONALIZADOS ATRAVÉS DE UM PROGRAMA GOVERNAMENTAL EM VILLAVICENCIO, NO PRIMEIRO SEMESTRE DE 2014}

Claudia Patricia Rodas Avellaneda, María del Pilar Angarita Díaz, Luis Francisco Nemocón Ramírez, Luis Alexys Pinzón Castro, Yenny Tatiana Robayo Herrera, Inés Leonilde Rodríguez Baquero, Rocío del Pilar González Sánchez

\section{Resumo}

Introdução. O processo de envelhecimento envolve mudanças fisiológicas que, juntamente com outros fatores, pode levar a doenças que afetam a cavidade oral. Cárie, doença periodontal e lesões das mucosas são as mais frequentes, e a sua presença, em grande parte, determina a qualidade de vida das pessoas idosas. Objetivo. Determinar o estado de saúde bucal de idosos institucionalizados através de um programa governamental de Villavicencio em três lares geriátricos.

Metodologia. Estudo descritivo de adultos idosos, onde foi recolhida a informação pessoal (dados e história médica) e os índices (COP-D, Silness e Löe modificado, CPITN e GOHAI) foram aplicados. Além disso, informações sobre as manifestações em tecidos moles e avaliação do edentulismo foram coletadas. Resultados. $48,5 \%$ da população apresentava edentulismo total e $51,5 \%$ ainda tinha dentes, mas com uma perda média de $18( \pm 7,2)$ dentes. Desta população, $85,7 \%$ dos idosos teve cárie, $74,1 \%$ apresentaram um fator de risco elevado da taxa de placa bacteriana e gengival, 33,3\% tinham doença periodontal. Além disso, verificou-se que $19 \%$ da população tinha uma lesão na mucosa e $76,5 \%$ tinham uma baixa percepção de qualidade de vida relacionada à saúde oral.

Conclusão. por meio de diferentes índices e ferramentas de avaliação, foi possível conhecer o estado bucal de idosos institucionalizados no âmbito de um programa governamental. Os resultados indicam a necessidade de implementação de estratégias e tratamentos para ajudar a melhorar a saúde bucal desta população vulnerável. 


\section{Introducción}

El aumento de la población mayor, en los últimos años, ha sido evidente a nivel mundial debido al descenso en las tasas de mortalidad y de fecundidad (1). Durante el último censo realizado en Colombia (2005) se estimó que la población de personas mayores de sesenta años ascendía a 3’815.453, de los cuales la mitad son de escasos recursos (2). El DANE, para el año 2020, proyectó 6’440.778 personas en este rango de edad (2), lo que obligaría al Estado y demás instituciones a establecer políticas y programas para fortalecer la atención a un grupo vulnerable que está en constante aumento. Según la Organización Mundial de la Salud, el proceso de envejecimiento es un curso biológico que tiene su propia dinámica (3), pero que también está sujeto al sentido que cada sociedad le otorga a este por medio del fomento y mantenimiento del bienestar durante la vejez (4).

El proceso de envejecimiento se hace evidente después de la sexta década de vida y es mayor y complejo a medida que el individuo aumenta de edad (5). El compromiso orgánico y psíquico implica tejidos, órganos, sistemas, autoestima, relaciones interpersonales y afectivas. A nivel biológico los cambios se deben a la acumulación de daños moleculares y celulares, que con el tiempo reducen gradualmente las reservas fisiológicas, aumentando el riesgo de contraer muchas enfermedades y disminuyendo, en general, la capacidad del individuo (4).

Las enfermedades de cavidad oral son algunas de las más frecuentes en los adultos mayores. Algunas de ellas son la caries, las enfermedades periodontales que conllevan a la pérdida de dientes, la xerostomía, las lesiones potencialmente malignas, el cáncer y otras lesiones de la mucosa oral (6). Estas enfermedades traen como consecuencia la disminución de la calidad de vida del adulto mayor debido a los problemas que genera en la masticación, deglución, trastorno del gusto y el olfato, y problemas de comunicación (7). Además, se ha demostrado que la salud oral deficiente, incrementa el riesgo de enfermedades cerebrovasculares, cardiovasculares y neumonías (8-11).

En Colombia, el último Estudio Nacional de Salud Bucal realizado en 2014 presentó las condiciones bucodentales de los adultos mayores en el rango de edad entre 65 y 79 años, con una prevalencia de caries en el $43,47 \%$ de la población, un índice COP (Cariados, Obturados, Perdidos) de 20,55, un valor promedio de pérdida de inserción a nivel periodontal de $3.15 \mathrm{~mm}$, una prevalencia de edentulismo total en el 37,71\% de la población, y de edentulismo parcial (al menos un diente perdido) en el $98,9 \%$. Además el $77,43 \%$ de la población edéntula en este rango de edad presenta algún tipo de prótesis (12).

Los centros residenciales para las personas mayores, son sitios destinados a la vivienda permanente o temporal donde se les brinda hospedaje, alimentación, recreación, actividades lúdicas, productivas, culturales y de cuidado integral, especialmente cuando presentan un alto índice de dependencia (13). Hacia este tipo de institución, la ley 1276 
de 2009 de Colombia, precisa que debe orientar los recursos del 30\% del recaudo de la estampilla para el bienestar de la Persona Mayor. Entre los hogares geriátricos o centros residenciales para el adulto mayor que apoya la alcaldía de Villavicencio, Meta, se encuentran la Obra Social San Camilo, la Casa del Divino Niño y San José. Los adultos mayores cuentan con un seguimiento médico básico brindado por el Sistema colombiano de salud, pero no cuentan con un seguimiento odontológico, ni con un diagnóstico y tratamiento de su estado bucodental. El objetivo principal de este estudio fue determinar el estado bucodental de las personas mayores institucionalizadas mediante un programa público en estos tres hogares geriátricos, a partir del uso de herramientas e instrumentos diagnósticos que permiten evidenciar las necesidades en salud bucodental de este grupo vulnerable.

\section{Materiales y Métodos}

Este estudio de tipo descriptivo, se realizó durante el primer semestre de 2014 con los adultos mayores de 65 años $(n=68)$ de los Centros de Protección Social para el adulto mayor, San Camilo, Divino Niño Jesús y San José de la ciudad de Villavicencio, Meta, que cumplían con los criterios de inclusión tales como: ser sostenidos por la alcaldía (debido al convenio docencia servicio No. 10-2011-021), tener un estado adecuado de salud física y mental, es decir que no presentaran una discapacidad mental y sensorial motora severa, y que firmaran el consentimiento informado para participar en el estudio. Para este estudio se tuvieron en cuenta las disposiciones internacionales de la declaración de Helsinki y lo dispuesto en la Resolución 8430 de 1993 del Ministerio de Salud de Colombia.

Además, se utilizaron instrumentos de recolección de la información que permitieron identificar variables categorizadas: datos personales, estado de salud general, enfermedades, hábitos, entre otros, e instrumentos para la medición de las condiciones bucodentales como el índice Comunitario de Necesidades de Tratamiento Periodontal (CPITN), el índice gingival y de placa de Silness y Löe modificado, el índice para evaluar la historia de caries dental de dientes Cariados, Obturados y Perdidos (COP), un instrumento para la selección de edentulismo parcial y total, el índice de Valoración de la Salud Oral geriátrica (GOHAI) y un instrumento para recolectar las manifestaciones orales más comunes en tejidos blandos en adultos mayores. Los índices CPITN, el índice gingival y de placa Silness y Löe y COP-D sólo se aplicaron a pacientes que presentaban dientes en boca y que pudieron ser examinados (Ver Figura 1).

Antes del examen oral de los adultos mayores, los estudiantes que optaban para el título de odontólogos fueron calibrados por parte de expertos en cada una de las áreas (Periodoncia, Rehabilitación y Patología oral). Para determinar el grado de acuerdo entre los integrantes se utilizó la prueba estadística del Coeficiente de Concordancia de Kendall (W), cuyo resultado $(0,75-0,85)$ permitió dar fiabilidad a la medición de los diferentes índices. 


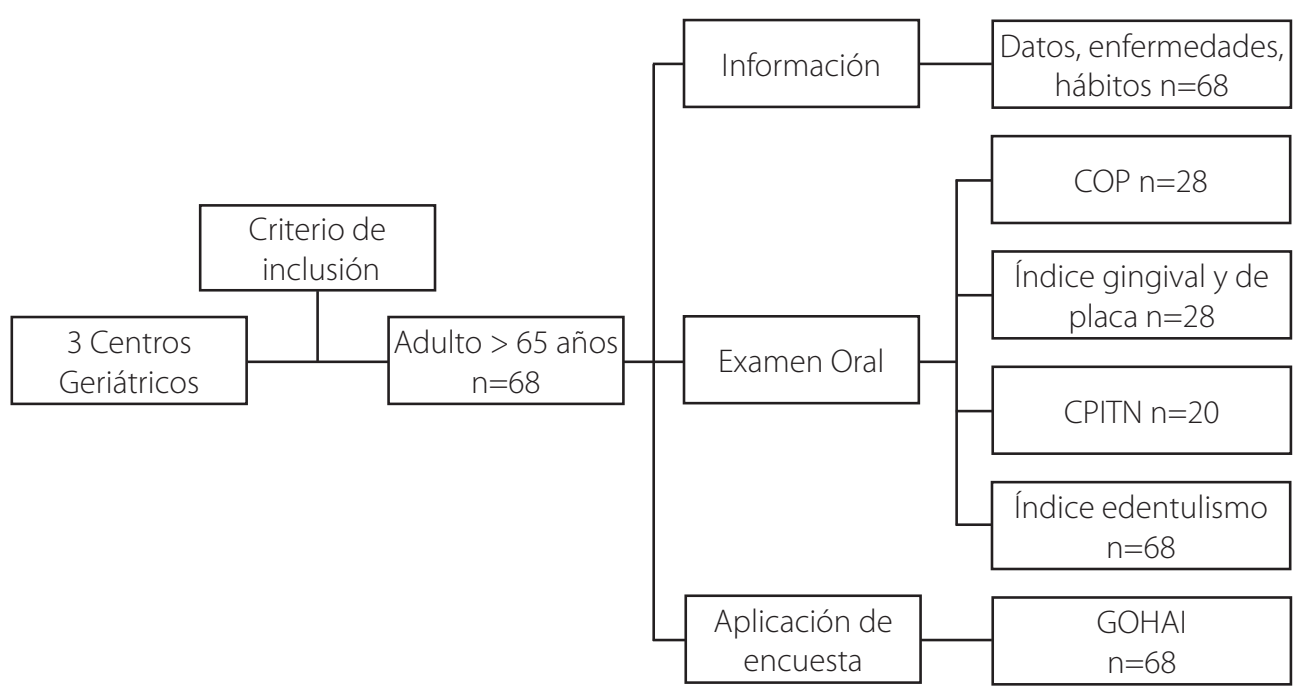

Figura 1. Esquema de la metodología para la recopilación de la información. Fuente: Elaboración propia sobre la metodología del estudio

Para el análisis de los datos se utilizó el programa SPSS 20.0, donde se realizó un análisis exploratorio de los datos (distribución de frecuencias, gráfico de barras) para las variables de forma univariada. Además se realizaron análisis descriptivos que determinaron proporciones, medias, medianas junto con las correspondientes medidas de dispersión (desviaciones estándar).

\section{Resultados}

\section{Características de la población evaluada}

El 48,5\% ( $n=33)$ de los adultos mayores pertenecían al Hogar San Camilo, el 27,9\% $(n=19)$ al Hogar San José y el
23,5\% ( $n=16)$ al Hogar Divino Niño. El promedio de edad de la población evaluada era de 78,6 $( \pm 9,5)$ años (mínimo 65 años, máximo 106 años) y la mayoría estaba conformada por el género masculino $(69,1 \%)$. Según la historia clínica, el 78\% de la población sufría alguna enfermedad, de estos el 53\% eran hombres y el 25\% mujeres. Entre las enfermedades más comunes, estaban las relacionada scon el sistema cardíaco (57\%) y nervioso (12\%). El $88 \%$ de la población tomaba algún tipo de medicamento. Respecto a los hábitos de higiene, el 79\% de las personas manifestaban hacer lavados de boca, dientes y prótesis con cepillo dental, el 74\% usaba crema dental y sólo el $7 \%$ seda dental (Tabla 1). 
Tabla 1. Descripción de la población valorada, antecedentes médicos y hábitos.

\begin{tabular}{lcc|c}
\hline \multicolumn{1}{c}{ Población } & Hombre & Mujer & Total \\
\hline Género & $70,6 \%(n=48)$ & $29,4 \%(n=20)$ & $100 \%(n=68)$ \\
Edad promedio & 78,5 años & 79 años & 78,6 años \\
Enfermedades sistémicas & $75 \%(n=36)$ & $85 \%(n=17)$ & $78 \%(n=53)$ \\
Sistema cardíaco & $52 \%(n=25)$ & $70 \%(n=14)$ & $57 \%(n=39)$ \\
Sistema respiratorio & $4,2 \%(n=2)$ & $10 \%(n=2)$ & $6 \%(n=4)$ \\
Sistema endocrino & $12,5 \%(n=6)$ & $5 \%(n=1)$ & $10 \%(n=7)$ \\
Sistema renal & $2,1 \%(n=1)$ & $5 \%(n=1)$ & $1 \%(n=2)$ \\
Sistema nervioso & $14,6 \%(n=7)$ & $5 \%(n=1)$ & $12 \%(n=8)$ \\
Sistema circulatorio & $2,1 \%(n=1)$ & $0 \%(n=0)$ & $1 \%(n=1)$ \\
Sistema gastrointestinal & $2,1 \%(n=2)$ & $5 \%(n=1)$ & $4 \%(n=3)$ \\
Sistema óseo & $6,3 \%(n=3)$ & $10 \%(n=2)$ & $7 \%(n=5)$ \\
Sistema tegumentario & $2,1 \%(n=1)$ & $0 \%(n=0)$ & $1 \%(n=1)$ \\
Consumo de medicamentos & $87,5 \%(n=42)$ & $90 \%(n=18)$ & $88 \%(n=60)$ \\
Hábitos de Higiene oral & & & $79 \%(n=5)$ \\
Uso de cepillo de dientes & $81,3 \%(n=39)$ & $75 \%(n=15)$ & $79 \%(n=54)$ \\
Uso de crema dental & $77,1 \%(n=37)$ & $70 \%(n=14)$ & $74 \%(n=2)$ \\
Uso de seda dental & $6,3 \%(n=3)$ & $10 \%(n)$ & \\
\hline
\end{tabular}

Fuente: Elaboración propia a partir de los resultados de la investigación

\section{Evaluación de Tejidos duros}

El índice COP sólo pudo ser tomado en 28 adultos mayores (20 hombres y 8 mujeres $)$, de estos el $85,7 \%(n=24)$ presentaban dientes cariados, el 7\% $(\mathrm{n}=2)$ presentaban dientes obturados, y el $100 \%(n=28)$ presentaban pérdida de dientes. El promedio de dientes cariados fue de $6,1( \pm 5,9)$, donde el 52\% $(n=15)$ de los adultos mayores presentaban más de 5 dientes cariados. El promedio de dientes perdidos era de $18( \pm 7,2)$ donde el $86 \%(n=25)$ de los adultos mayores parcialmente edéntulos presentaban más de 10 dientes perdidos. Finalmente, es importante mencionar que el promedio del total índice de COP-D fue de 24,89 $( \pm 4,6)$ (Tabla 2). 
INVESTIGACIONES ANDINA No. 33 Vol. 18

Tabla 2. Valoración COP-D, Índice de placa blanda e Índice de inflamación gingival.

\begin{tabular}{|c|c|c|c|c|c|c|}
\hline \multirow{2}{*}{$\begin{array}{c}\text { Examen } \\
\text { clínico oral }\end{array}$} & \multicolumn{2}{|c|}{ Hombre } & \multicolumn{2}{|c|}{ Mujer } & \multicolumn{2}{|c|}{ Total } \\
\hline & Porcentaje & Promedio & Porcentaje & Promedio & Porcentaje & Promedio \\
\hline $\begin{array}{l}\text { Dientes } \\
\text { cariados }\end{array}$ & $90 \%(n=18)$ & $5,5 \pm 5,5$ & $75 \%(n=6)$ & $3,5 \pm 2,6$ & $\begin{array}{l}85,7 \% \\
(n=24)\end{array}$ & $6,1 \pm 5,9$ \\
\hline $\begin{array}{l}\text { Dientes } \\
\text { obturados }\end{array}$ & $5 \%(n=1)$ & $0,37 \pm 1$ & $13 \%(n=1)$ & $0,1 \pm 0,35$ & $7 \%(n=2)$ & $0,14 \pm 0,59$ \\
\hline $\begin{array}{l}\text { Dientes } \\
\text { perdidos }\end{array}$ & $\begin{array}{c}100 \% \\
(n=20)\end{array}$ & $18,37 \pm 7,7$ & $100 \%(n=8)$ & $21,6 \pm 4,5$ & $\begin{array}{c}100 \% \\
(n=28)\end{array}$ & $18 \pm 7,2$ \\
\hline \multirow[t]{2}{*}{$\begin{array}{l}\text { Total } \\
\text { COP-D }\end{array}$} & NA & $24,25 \pm 6,2$ & NA & $25,2 \pm 2,5$ & NA & $24,89 \pm 4,59$ \\
\hline & $\begin{array}{c}100 \% \\
(n=21)\end{array}$ & $2 \pm 1$ & $100 \%(n=6)$ & $2,4 \pm 0,8$ & $\begin{array}{c}100 \% \\
(n=27)\end{array}$ & $2,1 \pm 1$ \\
\hline \multirow{3}{*}{$\begin{array}{l}\text { Índice de } \\
\text { Placa blanda }\end{array}$} & $\begin{array}{c}\text { FR alto: } \\
71,4 \%(n=15)\end{array}$ & $\begin{array}{c}\text { FR alto: } \\
2,64 \pm 0,5\end{array}$ & $\begin{array}{c}\text { FR alto: } \\
83,3 \%(n=5)\end{array}$ & $\begin{array}{l}\text { FR alto: } \\
2,72 \pm 0,4\end{array}$ & $\begin{array}{c}\text { FR alto: } \\
74 \%(n=20)\end{array}$ & $\begin{array}{l}\text { FR alto: } \\
2,7 \pm 0,45\end{array}$ \\
\hline & $\begin{array}{l}\text { FR. Medio: } \\
9,5 \%(n=2)\end{array}$ & $\begin{array}{c}\text { FR. Medio: } \\
0,9 \pm 0,1\end{array}$ & $\begin{array}{l}\text { FR. Medio: } \\
16,6 \% n=1\end{array}$ & $\begin{array}{c}\text { FR. Medio: } \\
1\end{array}$ & $\begin{array}{l}\text { FR. Medio: } \\
11,1 \% n=3)\end{array}$ & $\begin{array}{c}\text { FR. Medio: } \\
0,93 \pm 0,1\end{array}$ \\
\hline & $\begin{array}{l}\text { FR bajo: } \\
19 \%(n=4)\end{array}$ & $\begin{array}{l}\text { FR bajo: } \\
0,37 \pm 0,1\end{array}$ & FR bajo: 0 & FR bajo: 0 & $\begin{array}{c}\text { FR bajo: } \\
14,8 \%(n=4)\end{array}$ & $\begin{array}{l}\text { FR bajo: } \\
0,4 \pm 0,1\end{array}$ \\
\hline \multirow{4}{*}{$\begin{array}{l}\text { Índice } \\
\text { Gingival }\end{array}$} & $\begin{array}{c}100 \% \\
(n=21)\end{array}$ & $2 \pm 1$ & $100 \%(n=6)$ & $2,4 \pm 0,8$ & $\begin{array}{c}100 \% \\
(n=27)\end{array}$ & $2,1 \pm 1$ \\
\hline & $\begin{array}{c}\text { FR alto: } \\
71,4 \%(n=15)\end{array}$ & $\begin{array}{c}\text { FR alto: } \\
2,64 \pm 0,5\end{array}$ & $\begin{array}{c}\text { FR alto: } \\
83,3 \%(n=5)\end{array}$ & $\begin{array}{l}\text { FR alto: } \\
2,72 \pm 0,4\end{array}$ & $\begin{array}{c}\text { FR alto: } \\
74 \%(n=20)\end{array}$ & $\begin{array}{c}\text { FR alto: } \\
2,7 \pm 0,45\end{array}$ \\
\hline & $\begin{array}{l}\text { FR. Medio: } \\
9,5 \%(n=2)\end{array}$ & $\begin{array}{c}\text { FR. Medio: } \\
0,9 \pm 0,1\end{array}$ & $\begin{array}{l}\text { FR. Medio: } \\
16,6 \% n=1\end{array}$ & $\begin{array}{c}\text { FR. Medio: } \\
1\end{array}$ & $\begin{array}{l}\text { FR. Medio: } \\
11,1 \%(n=3)\end{array}$ & $\begin{array}{c}\text { FR. Medio: } \\
0,93 \pm 0,1\end{array}$ \\
\hline & $\begin{array}{l}\text { FR bajo: } \\
19 \%(n=4)\end{array}$ & $\begin{array}{l}\text { FR bajo: } \\
0,37 \pm 0,1\end{array}$ & FR bajo: 0 & FR bajo: 0 & $\begin{array}{c}\text { FR bajo: } \\
14,8 \%(n=4)\end{array}$ & $\begin{array}{l}\text { FR bajo: } \\
0,4 \pm 0,1\end{array}$ \\
\hline
\end{tabular}

† NA: No Aplica. \pm : Desviación estándar.

Fuente: Elaboración propia a partir de los resultados de la investigación.

Respecto a las necesidades de tratamiento, se detectó que el $55,17 \%$ $1632(\mathrm{n}=16)$ de los adultos mayores presentaban dientes con pérdida de estruc- tura pero recuperables en el maxilar superior, y el 52\% $(n=15)$ en el maxilar inferior. Además, se observó que el $17 \%(n=5)$ requerían valoración por 
endodoncia en dientes del maxilar superior y el $13,8 \%(n=4)$ en el maxilar inferior. El 58,6\% $(n=17)$ requerían valoración por periodoncia en el maxilar superior y el $65,5 \%(n=19)$ en el maxilar inferior.

\section{Evaluación placa dental, índice gingival y enfermedad periodontal}

En el índice de placa bacteriana y gingival se encontró que el $74 \%(n=20)$ de los 28 adultos mayores que conservaban dientes, presentaron un factor de riesgo alto con un promedio de índice de 2,7 $( \pm 0,45)$ y el $14,8 \%(n=4)$ un factor de riesgo bajo con un promedio de índice de $0,4( \pm 0,1)$. (Tabla 2 ).

De 21 personas mayores a quienes se les pudo aplicar el índice CPITN (Community Periodontal Index of Treatment Needs) por tener al menos dos dientes en algunos de los sextantes, el 95,2\% de la población requería algún tipo de tratamiento periodontal. Sólo había un adulto mayor del género femenino con tejido periodontal sano. El 38,1\% $(n=8)$ presentaron sangrado durante el sondaje, el 23,8\% $(n=5)$ presentaban placa calcificada y el 33,3\% $(n=7)$ presentaron bolsa de 4 a $5 \mathrm{~mm}$. (Tabla 3 ).

Tabla 3. Distribución estado del tejido periodontal de la población, según el índice CPITN.

\begin{tabular}{lcc|c}
\hline \multicolumn{1}{c|}{ Criterio } & Hombre & Mujer & Total \\
\hline Tejido periodontal sano & $0 \%(n=0)$ & $16,7 \%(n=1)$ & $4,8 \%(n=1)$ \\
Sangrado durante el sondaje & $40 \%(n=6)$ & $33,3 \%(n=2)$ & $38,1 \%(n=8)$ \\
Presencia de placa calcificada & $26,7 \%(n=4)$ & $16,7 \%(n=1)$ & $23,8 \%(n=5)$ \\
Bolsa de 4 a $5 \mathrm{~mm}$ & $33,3(n=5)$ & $33,3 \%(n=2)$ & $33,3(n=7)$ \\
\hline
\end{tabular}

Fuente: Elaboración propia a partir de los resultados de la investigación.

De 42 sextantes que pudieron ser valorados por tener más de dos dientes, el 90,4\% requerían de algún tipo de tratamiento. Se determinó que el tratamiento mayormente requerido fue la mejora de la higiene bucal $(42,8 \%)$ y la profilaxis (30,9\%). Esto se detectó con mayor frecuencia en el sextante medio inferior (62,5\%). Además se observó que el 16,8\% de los sextantes requerían tratamiento especial periodontal $(n=7)$. (Tabla 4). 
INVESTIGACIONES ANDINA No. 33 Vol. 18

Tabla 4. Distribución del índice CPITN por sextantes valorados.

\begin{tabular}{|c|c|c|c|c|c|c|c|}
\hline $\begin{array}{l}\text { Tratamiento } \\
\text { requerido }\end{array}$ & $\begin{array}{c}\text { Sextante } \\
1\end{array}$ & $\begin{array}{c}\text { Sextante } \\
2\end{array}$ & $\begin{array}{c}\text { Sextante } \\
3\end{array}$ & $\begin{array}{c}\text { Sextante } \\
4\end{array}$ & $\begin{array}{c}\text { Sextante } \\
5\end{array}$ & $\begin{array}{c}\text { Sextante } \\
6\end{array}$ & $\begin{array}{c}\text { Total } \\
\text { sextante }\end{array}$ \\
\hline $\begin{array}{l}0=\text { no } \\
\text { necesita } \\
\text { tratamiento }\end{array}$ & $0(n=0)$ & $0(n=0)$ & $0(n=0)$ & $\begin{array}{l}33,3 \% \\
(n=1)\end{array}$ & $\begin{array}{l}12,5 \% \\
(n=2)\end{array}$ & $\begin{array}{l}50 \% \\
(n=1)\end{array}$ & $\begin{array}{l}9,5 \% \\
(n=4)\end{array}$ \\
\hline $\begin{array}{l}\text { 1=Mejorar } \\
\text { la higiene } \\
\text { bucal }\end{array}$ & $\begin{array}{l}28,6 \% \\
(n=2)\end{array}$ & $\begin{array}{l}57,1 \% \\
(n=4)\end{array}$ & $\begin{array}{l}28,6 \% \\
(n=2)\end{array}$ & $0(n=0)$ & $\begin{array}{l}62,5 \% \\
(n=10)\end{array}$ & $0(n=0)$ & $\begin{array}{l}42,8 \% \\
(n=18)\end{array}$ \\
\hline $\begin{array}{l}2=\text { Mejorar } \\
\text { higiene } \\
\text { bucal y } \\
\text { raspaje } \\
\text { profesional }\end{array}$ & $\begin{array}{l}57,1 \% \\
(n=4)\end{array}$ & $\begin{array}{l}14,3 \% \\
(n=1)\end{array}$ & $\begin{array}{l}57,1 \% \\
(n=4)\end{array}$ & $\begin{array}{l}33,3 \% \\
(n=1)\end{array}$ & $\begin{array}{l}12,5 \% \\
(n=2)\end{array}$ & $\begin{array}{l}50 \% \\
(n=1)\end{array}$ & $\begin{array}{l}30,9 \% \\
(n=13)\end{array}$ \\
\hline $\begin{array}{l}3=\text { Mejorar } \\
\text { higiene } \\
\text { bucal y } \\
\text { tratamiento } \\
\text { especial }\end{array}$ & $\begin{array}{l}14,3 \% \\
(n=1)\end{array}$ & $\begin{array}{l}28,6 \% \\
(n=2)\end{array}$ & $\begin{array}{l}14,3 \% \\
(n=1)\end{array}$ & $\begin{array}{l}33,3 \% \\
(n=1)\end{array}$ & $\begin{array}{l}12,5 \% \\
(n=2)\end{array}$ & $0(n=0)$ & $\begin{array}{l}16,7 \% \\
(n=7)\end{array}$ \\
\hline Total & $\begin{array}{l}100 \% \\
(n=7)\end{array}$ & $\begin{array}{l}100 \% \\
(n=7)\end{array}$ & $\begin{array}{l}100 \% \\
(n=7)\end{array}$ & $\begin{array}{l}100 \% \\
(n=3)\end{array}$ & $\begin{array}{c}100 \% \\
(n=16)\end{array}$ & $\begin{array}{l}100 \% \\
(n=2)\end{array}$ & $\begin{array}{c}100 \% \\
(n=42)\end{array}$ \\
\hline
\end{tabular}

Fuente: Elaboración propia a partir de los resultados de la investigación.

\section{Edentulismo y prótesis}

El $100 \%$ de la población ( $\mathrm{n}=68$ ) había perdido dientes (mínimo 4, máximo 28), con un promedio total de 23,3 $\pm 6,9$ dientes perdidos. Del total de la población examinada, el $48,5 \%(n=33)$ eran totalmente edéntulos y el $51,5 \%(n=35)$ eran parcialmente edéntulos con un promedio de dientes en boca de 4,7 $\pm 6,9$ (Tabla 5). El edentulismo fue mayor en el maxilar superior (60,4\%). Según la clasificación de Kennedy el 2,9\% de los adultos parcialmente edéntulos en el maxilar superior, eran de clase I, el 1,5\% eran de clase II, el 7,3\% de clase III y el 5,8\% de clase IV. En el maxilar inferior predominaba la clase I con $22,1 \%$, las clases II y III con $1,5 \%$, y la clase IV con 2,9\% (Figura 2). Respecto a la clasificación de Seibert para rebordes alveolares predominaba la clase III, en el maxilar superior e inferior. 
Tabla 5. Descripción edentulismo en la población valorada.

\begin{tabular}{|c|c|c|c|c|c|c|}
\hline \multirow{2}{*}{$\begin{array}{c}\text { Examen } \\
\text { clínico oral }\end{array}$} & \multicolumn{2}{|c|}{ Hombre } & \multicolumn{2}{|c|}{ Mujer } & \multicolumn{2}{|c|}{ Total } \\
\hline & Porcentaje & Promedio & Porcentaje & Promedio & Porcentaje & Promedio \\
\hline $\begin{array}{l}\text { Adultos con } \\
\text { al menos } \\
\text { un diente } \\
\text { perdido }\end{array}$ & $\begin{array}{c}100 \% \\
(n=48)\end{array}$ & $\begin{array}{c}22,8 \pm 7,6 \\
\text { dientes } \\
\text { perdidos }\end{array}$ & $00 \%(n=20)$ & $\begin{array}{c}24,6 \pm 4,6 \\
\text { dientes } \\
\text { perdidos }\end{array}$ & $\begin{array}{c}100 \% \\
(n=68)\end{array}$ & $\begin{array}{c}23,3 \pm 6,9 \\
\text { dientes } \\
\text { perdidos }\end{array}$ \\
\hline $\begin{array}{l}\text { Totalmente } \\
\text { edéntulos }\end{array}$ & $\begin{array}{l}52,1 \% \\
(n=25)\end{array}$ & NA & $40 \%(n=8)$ & NA & $\begin{array}{l}48.5 \% \\
(n=33)\end{array}$ & NA \\
\hline $\begin{array}{l}\text { Parcialmente } \\
\text { edéntulos }\end{array}$ & $\begin{array}{l}47,9 \% \\
(n=23)\end{array}$ & $\begin{array}{c}5 \pm 7,5 \\
\text { dientes en } \\
\text { boca }\end{array}$ & $60 \%(n=12)$ & $\begin{array}{c}3,7 \pm 5,1 \\
\text { dientes en } \\
\text { boca }\end{array}$ & $\begin{array}{l}51,5 \% \\
(n=35)\end{array}$ & $\begin{array}{c}\text { 4,7 } \pm 6,9 \\
\text { dientes en } \\
\text { boca }\end{array}$ \\
\hline
\end{tabular}

$\dagger$ NA: No Aplica. \pm : Desviación estándar.

Fuente: Elaboración propia a partir de los resultados de la investigación.

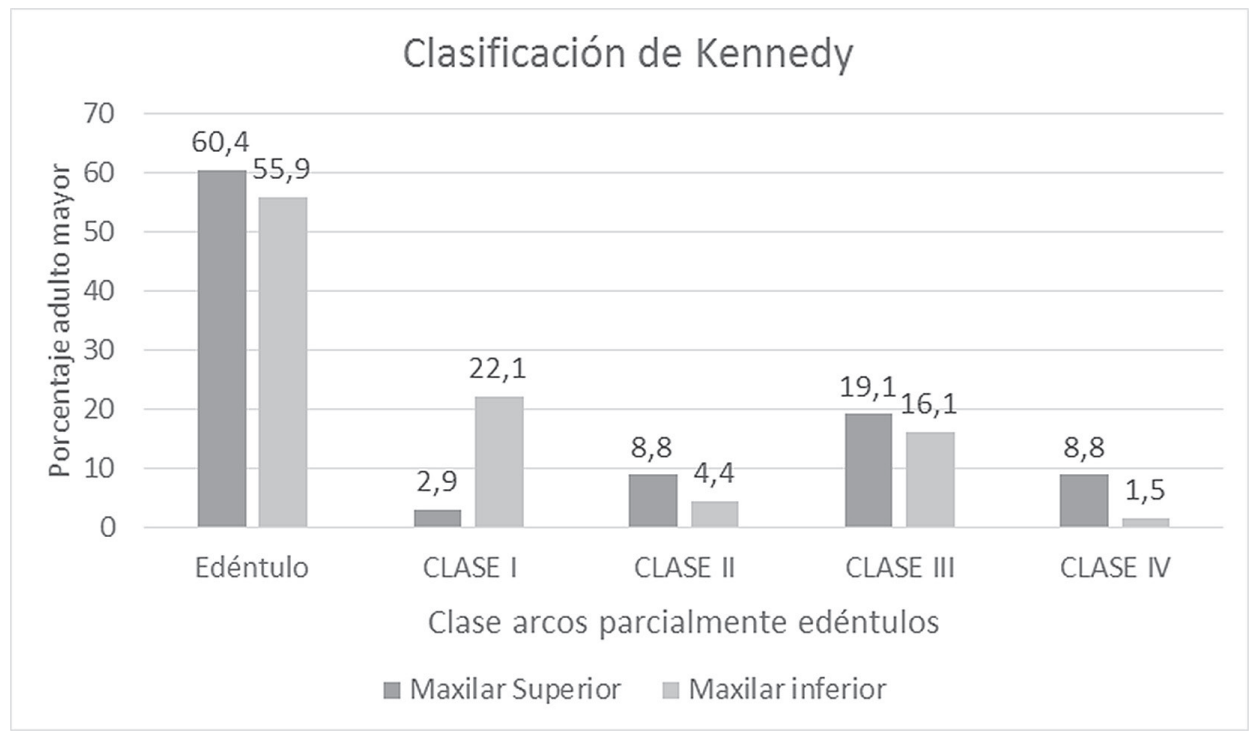

Figura 2. Distribución adultos mayores edéntulos y parcialmente edéntulos según la clasificación Kennedy, de acuerdo al maxilar superior e inferior.

Fuente: Elaboración propia a partir de los resultados de la investigación. 
Clase I: Áreas edéntulas bilaterales posteriores a los dientes naturales. Clase II: Área edéntula unilateral, posterior a los dientes naturales remanentes. Clase III: Área edéntula uni- lateral con dientes remanentes hacia anterior y posterior. Clase IV: Área edéntula única, bilateral que atraviesa la línea media, anterior a los dientes remanentes.

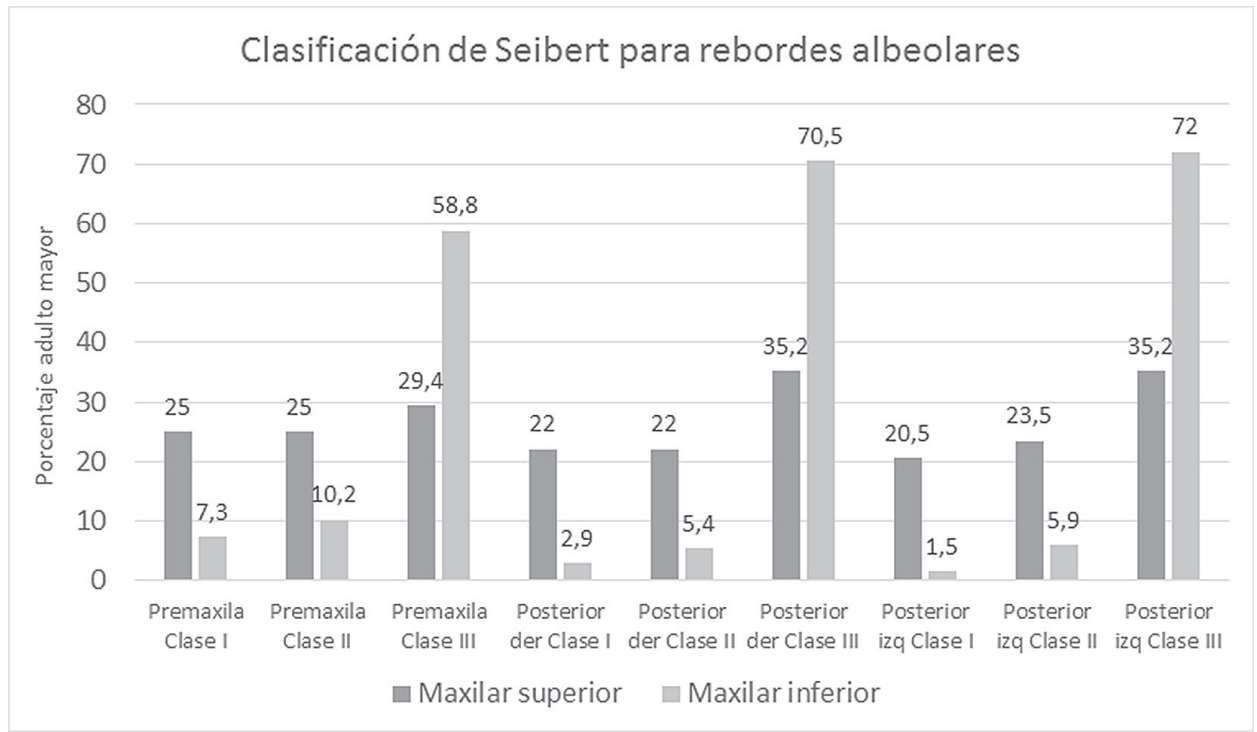

Figura 3. Clasificación de Seibert para rebordes albeolares

Fuente: Elaboración propia a partir de los resultados de la investigación.

\section{Evaluación de prótesis}

Respecto a la aparatología en boca, el $47 \%$ de las personas $(n=32)$ presentaban prótesis. El 1,5\% $(n=1)$ de los adultos mayores presentaban prótesis fija (inferior), el $14,7 \%$ prótesis parcial removible $(n=10)$ y el $39,7 \%(n=27)$ prótesis total removible. De los 68 adultos mayores examinados, el $77,94 \%(n=53)$ evidenciaron la necesidad de prótesis o cambio de esta. El 16,2\% ( $\mathrm{n}=11)$ necesitaban prótesis parcial removible mucosoportada en el maxilar superior y $22,1 \%(n=15)$ en el inferior. El 2,9\% $(n=2)$ necesitaba prótesis removible dentomucosoportada en el maxilar superior y 4,4\% $(n=3)$ en el inferior y por último el $58,8 \%(\mathrm{n}=40)$ requerían prótesis total mucosoportada en el maxilar superior y el 51,5\% $(n=35)$ en el inferior (Tabla 6). 
Tabla 6. Valoración del estado protésico

\begin{tabular}{|c|c|c|c|}
\hline Prótesis & Hombre & Mujer & Total \\
\hline Prótesis fija & $2 \%(n=1)$ & $0 \%(n=0)$ & $1,5 \%(n=1)$ \\
\hline Inferior & $2 \%(n=1)$ & $0 \%(n=0)$ & $1,5 \%(n=1)$ \\
\hline Prótesis parcial removible & $12,5 \%(n=6)$ & $20 \%(n=4)$ & $14,7 \%(n=10)$ \\
\hline Superior & $8,3 \%(n=4)$ & $5 \%(n=1)$ & $7,4 \%(n=5)$ \\
\hline Inferior & $4,1 \%(n=2)$ & $10 \%(n=2)$ & $5,9 \%(n=4)$ \\
\hline Prótesis total removible & $41,6 \%(n=20)$ & $35 \%(n=7)$ & $39,7 \%(n=27)$ \\
\hline Superior & $37,5 \%(n=18)$ & $20 \%(n=4)$ & $33,8 \%(n=23)$ \\
\hline Inferior & $31,3 \%(n=15)$ & $10 \%(n=2)$ & $25 \%(n=17)$ \\
\hline \multicolumn{4}{|c|}{ Necesidad de cambio o de elaboración de prótesis } \\
\hline $\begin{array}{l}\text { Necesidad de prótesis o } \\
\text { cambio de esta }\end{array}$ & $79,2 \%(n=38)$ & $75 \%(n=15)$ & $77,94 \%(n=53)$ \\
\hline $\begin{array}{l}\text { Prótesis parcial removible } \\
\text { mucosuportada superior }\end{array}$ & $18,8 \%(n=9)$ & $10 \%(n=2)$ & $16,2 \%(n=11)$ \\
\hline $\begin{array}{l}\text { Prótesis removible } \\
\text { dentomucosuportada } \\
\text { superior }\end{array}$ & $2 \%(n=1)$ & $5 \%(n=1)$ & $2,9 \%(n=2)$ \\
\hline Prótesis total superior & $58,3 \%(n=28)$ & $60 \%(n=12)$ & $58,8 \%(n=40)$ \\
\hline $\begin{array}{l}\text { Prótesis parcial removible } \\
\text { mucosuportada inferior }\end{array}$ & $22,9 \%(n=11)$ & $20 \%(n=4)$ & $22.1 \%(n=15)$ \\
\hline $\begin{array}{l}\text { Prótesis removible } \\
\text { dentomucosuportada inferior }\end{array}$ & $2 \%(n=1)$ & $10 \%(n=2)$ & $4,4 \%(n=3)$ \\
\hline Prótesis total inferior & $54,2 \%(n=26)$ & $45 \%(n=9)$ & $51,5 \%(n=35)$ \\
\hline
\end{tabular}

Fuente: Elaboración propia a partir de los resultados de la investigación.

\section{Evaluación de Tejidos blandos}

Se identificó que el 19\% $(n=13)$ de la población valorada presentaban manifestaciones orales entre las que se des- tacaban las venas varicosas con un $8,8 \%$ $(n=6)$ distribuidos entre hombres $(n=4)$ y mujeres $(n=2)$, la lengua saburral (3) y las aftas menores (2), observadas solo en hombres. (Figura 4). 


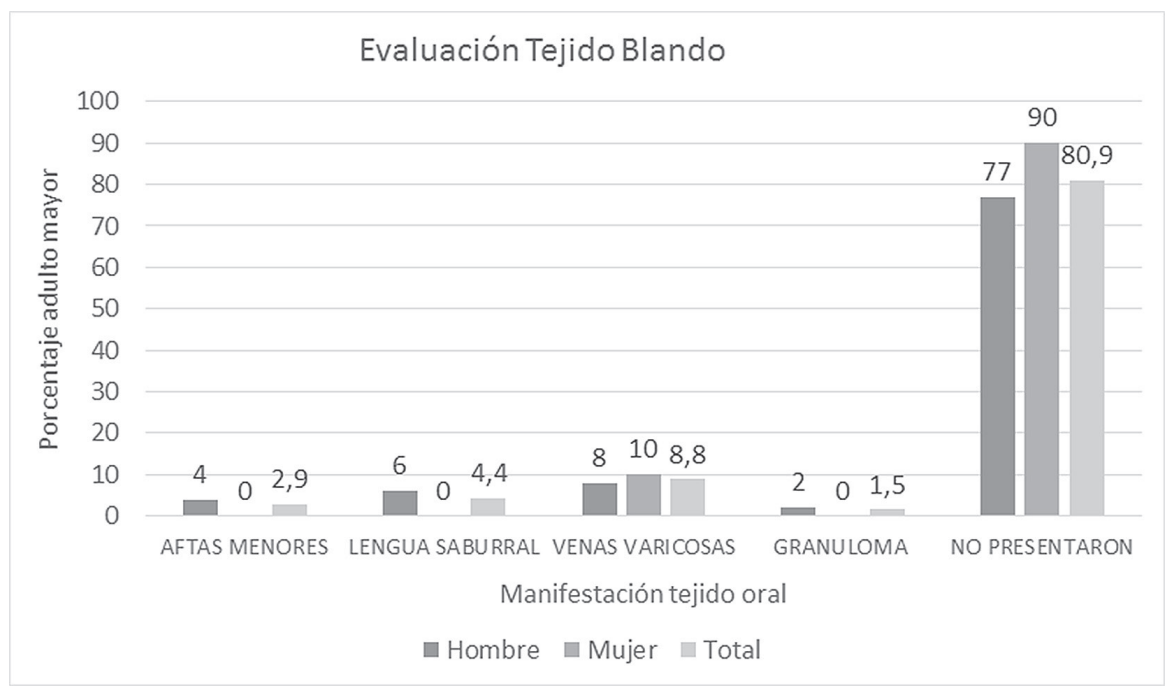

Figura 4. Manifestaciones orales en tejidos blandos

Fuente: Elaboración propia a partir de los resultados de la investigación.

\section{Índice de GOHAI}

La puntuación GOHAI promedio para el índice GOHAI (Índice de Salud oral Geriátrico de su sigla en inglés Geriatric/General Health Assesment Index) fue de $43,5( \pm 8,2)$, lo que corresponde a una baja autopercepción de la calidad de vida que está relacionada con un estado pobre de salud bucal. Los resultados indicaron que el 76,5\% $(\mathrm{n}=52)$ tienen una baja autopercepción de calidad de vida, el 16,2\% $(n=11)$ tienen una percepción moderada y sólo el $7,3 \%(n=5)$ tienen una percepción alta (Tabla 7).

Tabla 7. Nivel de autopercepción de la salud bucodental del adulto mayor, índice GOHAI

\begin{tabular}{|c|c|c|c|c|c|c|}
\hline \multirow{2}{*}{$\begin{array}{l}\text { Índice } \\
\text { GOHAI }\end{array}$} & \multicolumn{2}{|c|}{ Hombre } & \multicolumn{2}{|c|}{ Mujer } & \multicolumn{2}{|c|}{ Total } \\
\hline & Porcentaje & Promedio & Porcentaje & Promedio & Porcentaje & Promedio \\
\hline TOTAL & $\begin{array}{c}100 \% \\
(n=48)\end{array}$ & $45,25 \pm 8,9$ & $\begin{array}{c}100 \% \\
(n=20)\end{array}$ & $41,7 \pm 8,22$ & $\begin{array}{c}100 \% \\
(n=68)\end{array}$ & $43,5 \pm 8,2$ \\
\hline $\begin{array}{l}\text { Autopercepción } \\
\text { Alta }\end{array}$ & $\begin{array}{l}6,25 \% \\
(n=3)\end{array}$ & $59 \pm 1$ & $10 \%(n=2)$ & $58,5 \pm 2,1$ & $7,3 \%(n=5)$ & $58.8 \pm 1,3$ \\
\hline $\begin{array}{l}\text { Autopercepción } \\
\text { Moderada }\end{array}$ & $\begin{array}{l}20,8 \% \\
(n=10)\end{array}$ & $54,6 \pm 1,8$ & $5 \%(n=1)$ & 52 & $\begin{array}{l}16,2 \% \\
(n=11)\end{array}$ & $54,4 \pm 1,9$ \\
\hline $\begin{array}{l}\text { Autopercepción } \\
\text { Baja }\end{array}$ & $\begin{array}{c}73 \% \\
(n=35)\end{array}$ & $41,4 \pm 7,2$ & $\begin{array}{c}85 \% \\
(n=17)\end{array}$ & $39,1 \pm 5,6$ & $\begin{array}{l}76,5 \% \\
(n=52)\end{array}$ & $40,6 \pm 6,7$ \\
\hline
\end{tabular}

$\dagger \pm$ : Desviación estándar. $\ddagger$ Categorías GOHAI: Nivel alto: 57-60. Nivel moderado: 51-56. Nivel bajo: 50 o menos.

Fuente: Elaboración propia a partir de los resultados de la investigación. 
Al analizar las dimensiones evaluadas por el índice GOHAI, que relacionan la salud bucal con la calidad de vida, se encontró que la función psicosocial en la pregunta relacionada con la autoimagen, el $35,3 \%$ de las personas mayores contestaron que nunca se han sentido contentos como se ven los dientes cuando se miran al espejo y en la pregunta relacionada con la preocupación acerca de la salud oral, el 32,4\% contestaron que siempre se han sentido preocupados o se han dado cuenta que los dientes no están bien. Respecto a la función física, que comprende aspectos como comer, tragar o hablar, el 32,4\% nunca ha pasado bien los alimentos y el 17,6\% contestaron que algunas veces (Tabla 8).

Tabla 8. Índice GOHAI por dimensiones

\begin{tabular}{|c|c|c|c|c|c|}
\hline \multicolumn{6}{|c|}{ Índice de GOHAI } \\
\hline Dimensiones & 1 & 2 & 3 & 4 & 5 \\
\hline Función Física & Siempre & Frecuentemente & $\begin{array}{c}\text { Algunas } \\
\text { Veces }\end{array}$ & Rara Vez & Nunca \\
\hline $\begin{array}{l}\text { 1.¿Cuántas veces ha } \\
\text { tenido que comer } \\
\text { menos o cambiar de } \\
\text { comida por culpa } \\
\text { de sus dientes o } \\
\text { dentaduras? }\end{array}$ & $\begin{array}{l}17,6 \% \\
(n=12)\end{array}$ & $\begin{array}{l}16,2 \% \\
(n=11)\end{array}$ & $10,3 \%(n=7)$ & $13,2 \%(n=9)$ & $42,6(n=29)$ \\
\hline $\begin{array}{l}\text { 2. ¿Cuántas veces ha } \\
\text { tenido problemas } \\
\text { al masticar comidas } \\
\text { como la carne o las } \\
\text { manzanas? }\end{array}$ & $\begin{array}{l}23,5 \% \\
(n=16)\end{array}$ & $19, \%(n=13)$ & $\begin{array}{l}14,7 \% \\
(n=10)\end{array}$ & $8,8 \%(n=6)$ & $\begin{array}{l}33,8 \% \\
(n=23)\end{array}$ \\
\hline $\begin{array}{l}\text { 3. ¿Cuántas veces } \\
\text { ha pasado bien los } \\
\text { alimentos? }\end{array}$ & $\begin{array}{l}44,1 \% \\
(n=30)\end{array}$ & $5,4 \%(n=4)$ & $\begin{array}{l}17,6 \% \\
(n=12)\end{array}$ & 0 & $\begin{array}{l}32,4 \% \\
(n=22)\end{array}$ \\
\hline $\begin{array}{l}\text { 4.¿Cuántas veces no } \\
\text { ha podido usted } \\
\text { hablar bien por } \\
\text { culpa de sus dientes } \\
\text { o dentaduras? }\end{array}$ & $\begin{array}{l}11,8 \% \\
(n=8)\end{array}$ & $\begin{array}{c}14,7 \% \\
(n=10)\end{array}$ & $11,8 \%(n=8)$ & $11,8 \%(n=8)$ & $\begin{array}{l}50,0 \% \\
(n=34)\end{array}$ \\
\hline $\begin{array}{l}\text { 5. ¿Cuántas veces } \\
\text { no ha podido comer } \\
\text { las cosas que usted } \\
\text { quería sin tener } \\
\text { ninguna molestia? }\end{array}$ & $\begin{array}{l}14,7 \% \\
(n=10)\end{array}$ & $11,8 \%(n=8)$ & $\begin{array}{l}17,6 \% \\
(n=12)\end{array}$ & $\begin{array}{l}20,6 \% \\
(n=14)\end{array}$ & $\begin{array}{l}35,3 \% \\
(n=24)\end{array}$ \\
\hline Función psicosocial & & & & & \\
\hline $\begin{array}{l}\text { 6. ¿Cuántas veces no } \\
\text { ha querido salir a la } \\
\text { calle o hablar con la } \\
\text { gente por culpa de sus } \\
\text { dientes o dentaduras? }\end{array}$ & $\begin{array}{l}16,2 \% \\
(n=11)\end{array}$ & $5,9 \%(n=4)$ & $7,4 \%(n=5)$ & $10,3 \%(n=7)$ & $\begin{array}{l}60,3 \% \\
(n=41)\end{array}$ \\
\hline
\end{tabular}


Continuación Tabla 8. Índice GOHAI por dimensiones

\begin{tabular}{|c|c|c|c|c|c|}
\hline \multicolumn{6}{|c|}{ Índice de GOHAI } \\
\hline Dimensiones & 1 & 2 & 3 & 4 & 5 \\
\hline Función psicosocial & Siempre & Frecuentemente & $\begin{array}{c}\text { Algunas } \\
\text { Veces }\end{array}$ & Rara Vez & Nunca \\
\hline $\begin{array}{l}\text { 7. ¿Cuándo usted } \\
\text { se mira al espejo, } \\
\text { cuántas veces ha } \\
\text { estado contento de } \\
\text { cómo se ven sus } \\
\text { dientes? }\end{array}$ & $\begin{array}{l}35,3 \% \\
(n=24)\end{array}$ & $11,8 \%(n=8)$ & $11,8 \%(n=8)$ & $\begin{array}{l}5,9 \% \\
(n=4)\end{array}$ & $\begin{array}{l}35,3 \% \\
(n=24)\end{array}$ \\
\hline $\begin{array}{l}\text { 9. ¿Cuántas veces ha } \\
\text { estado preocupado } \\
\text { o se ha dado cuenta } \\
\text { de que sus dientes } \\
\text { o su dentadura no } \\
\text { están bien? }\end{array}$ & $\begin{array}{l}32,4 \% \\
(n=22)\end{array}$ & $8,8 \%(n=6)$ & $13,2 \%(n=9)$ & $\begin{array}{l}10,3 \% \\
(n=7)\end{array}$ & $\begin{array}{l}35,3 \% \\
(n=24)\end{array}$ \\
\hline $\begin{array}{l}\text { 10. ¿Cuántas veces } \\
\text { se ha puesto } \\
\text { nervioso por los } \\
\text { problemas de sus } \\
\text { dientes o de su } \\
\text { dentadura? }\end{array}$ & $\begin{array}{l}10,3 \% \\
(n=7)\end{array}$ & $5,9(n=4)$ & $\begin{array}{l}14,7 \% \\
(n=10)\end{array}$ & $\begin{array}{l}8,8 \% \\
(n=6)\end{array}$ & $\begin{array}{l}60,3 \% \\
(n=41)\end{array}$ \\
\hline $\begin{array}{l}\text { 11. ¿Cuántas veces } \\
\text { no ha comido a } \\
\text { gusto delante de } \\
\text { otras personas por } \\
\text { culpa de sus dientes } \\
\text { o dentadura? }\end{array}$ & $\begin{array}{l}13,2 \% \\
(n=9)\end{array}$ & $10,3 \%(n=7)$ & $\begin{array}{l}17,6 \% \\
(n=12)\end{array}$ & $13,2 \%(n=9)$ & $\begin{array}{l}45,6 \% \\
(n=31)\end{array}$ \\
\hline \multicolumn{6}{|l|}{ Función dolor } \\
\hline $\begin{array}{l}\text { 8. ¿Cuántas veces ha } \\
\text { tenido que utilizar } \\
\text { algún medicamento } \\
\text { para aliviar el dolor } \\
\text { de sus dientes o } \\
\text { las molestias en su } \\
\text { boca? }\end{array}$ & $\begin{array}{l}2,9 \% \\
(n=2)\end{array}$ & $5,9 \%(n=4)$ & $\begin{array}{c}14,7 \% \\
(n=10)\end{array}$ & $\begin{array}{l}10,3 \% \\
(n=7)\end{array}$ & $\begin{array}{l}66,2 \% \\
(n=45)\end{array}$ \\
\hline $\begin{array}{l}\text { 12. ¿Cuántas veces } \\
\text { ha tenido molestias } \\
\text { o dolor en sus } \\
\text { dientes por el frío, } \\
\text { el calor o las cosas } \\
\text { dulces? }\end{array}$ & $\begin{array}{l}4,4 \% \\
(n=4\end{array}$ & $2,9 \%(n=2)$ & $\begin{array}{l}19,1 \% \\
(n=13)\end{array}$ & $\begin{array}{l}4,4 \% \\
(n=3)\end{array}$ & $\begin{array}{l}69,1 \% \\
(n=47)\end{array}$ \\
\hline
\end{tabular}




\section{Discusión}

Este estudio partió con el propósito de conocer las condiciones bucodentales de adultos mayores sostenidos por un programa público en tres centros geriátricos. El $78 \%$ de la población presentaban diferentes enfermedades sistémicas, donde predominaban las cardiacas (57\%), nerviosas (12\%) y endocrinas (10\%) y el $88 \%$ de la población consumía distintos tipos de medicamentos. Algunos autores mencionan la relación entre las enfermedades sistémicas, el efecto indeseable de los medicamentos y otros factores (higiene, culturales, sociales, etc.) con el estado oral de los adultos mayores $(16)(18)(19)(20)$.

Entre los resultados encontrados en este estudio, el $48,5 \%$ de los adultos mayores eran totalmente edéntulos y el 51,5\% eran parcialmente edéntulos. De la población que aún conservaba dientes, el $85 \%$ presentaba caries, el $74,1 \%$ presentaba un factor de riesgo alto en el índice de placa bacteriana y gingival, el 33,3\% presentaba enfermedad periodontal. Además, se detectó en toda la población valorada, que el 76,5\% tenían una baja autopercepción de la salud oral según el índice GOHAI. Aunque la caries, la enfermedad periodontal, el edentulismo y la baja valoración de la salud oral geriátrica son frecuentes en la salud bucal de los adultos mayores, el establecimiento de medidas adecuadas disminuirán estos índices (4)(14).

De acuerdo a toda la población valorada en este estudio, el 41,2\% de la población presenta caries, resultado que es similar al presentado por el ENSAB en 2014, donde el 43,5\% de la población en el rango de edad entre 65-79 años, la presenta (12). El valor promedio de dientes cariados en este estudio fue de 6,1, siendo más alto que el observado por Bermúdez et $a l$, en población institucionalizada (6096 años) del área metropolitana de Bucaramanga, Colombia, donde encontraron un promedio de 3 dientes cariados (21). Además, se detectó que el promedio de dientes cariados fue más alto en hombres $(5,5)$ que en mujeres $(3,5)$, lo cual fue similar al estudio realizado por Esquivel y Jiménez, en una población de adultos mayores que asisten a una casa de descanso (60-92 años) en Tlalnepantla, México, donde también reportaron un mayor número de dientes cariados en hombres $(4,33)$ que en mujeres $(3,48)(22)$.

La población valorada para el índice COP-D, presentaron pérdida de dientes con un promedio de 18 dientes, siendo el valor más alto en mujeres $(21,6)$ que en hombres $(18,4)$. Esto también se observó en el estudio de Esquivel y Jiménez, donde las mujeres presentaban un mayor número de dientes perdidos $(15,6)$ que los hombres $(12,8)$ (22). En este estudio el componente de dientes perdidos elevó la cifra del índice COP-D, valor promedio que estuvo en 24,89 . El índice COP-D, es un indicador que aumenta con la edad haciéndose evidente en diferentes estudios a nivel nacional y mundial (12)(19)(22-25). Los resultados del presente estudio fueron más bajos que el estudio realizado por Bermúdez et al, en población institucionalizada, donde el promedio de dientes perdidos fue de 25 y el COP-D 27 (21); más altos que en el ENSAB IV (COP-D de 20,55) (12) y el realizado por Joya y Rodríguez en 
la ciudad de Manizales (COP-D de 23) (19) y similares al obtenido en la región de Maule, Chile, en adultos mayores entre 65 y 74 años, quienes presentaron un promedio de dientes perdidos de 22,4 $y$ un índice COP-D de 25,7 (26). Una revisión realizada por Mariño en 1994, presentó que el índice COP-D en adultos mayores de Venezuela, Chile, Costa Rica y Brasil mostraron promedios en un rango de 19,1 a 27,2, siendo el componente de dientes perdidos el que eleva el índice (27). Por tanto, la experiencia de caries de este estudio, está dentro del rango determinado en población mayor latinoamericana.

El índice de placa blanda y el índice gingival, determinaron que gran parte de la población examinada, presentaban un factor de riesgo alto (74\%) con un promedio del índice de 2,7 indicando la presencia de placa abundante en el surco gingival e inflamación gingival grave. Así mismo el Índice de Necesidades de Tratamiento Periodontal (CPITN) no indicó que el 95,2\% de la población presentaba algún problema periodontal con diferentes grados de severidad. El 33,3\% presentaban bolsa de 4 a $5 \mathrm{~mm}$ requiriendo tratamiento periodontal especializado, el $23,8 \%$ presentó placa calcificada donde se hacía necesario el raspaje profesional y el $38,1 \%$ sangrado durante el sondaje donde era necesario educación en salud y profilaxis. Esto se confirmó también al analizar los resultados por los sextantes valorados. Los resultados del presente estudio son similares a los obtenidos en otros estudios que utilizaron el mismo índice, como el de Bermúdez et al, donde detectaron que el 93,4\% de la población evaluada requería algún tipo de tratamiento periodontal, aunque existía variaciones en cuanto a necesidades de tratamiento, ya que el $63 \%$ requería tratamiento especializado, $21,7 \%$ raspaje profesional, 8,7\% educación en salud y profilaxis (21). Mariño y Giacam, observaron la necesidad de tratamiento en el $100 \%$ de la población: $30 \%$ requería tratamiento especializado, $68,4 \%$ raspaje profesional, 1,5\% educación en salud y profilaxis (26). Y Joya y Quintero, 2015, encontraron que el $95 \%$ de los adultos mayores requerían tratamiento, distribuidos en: $41 \%$ tratamiento especializado, $31 \%$ raspaje profesional, $23 \%$ educación en salud y profilaxis (19).

En este estudio, a pesar de que la gran mayoría de adultos mayores que manifestaron mantener higiene oral mediante el uso de cepillo de dientes (79\%) y uso de crema dental (74\%), los índices de placa y el de necesidad de tratamiento periodontal, indican una mala higiene probablemente por la ausencia en el conocimiento de las técnicas adecuadas y en algunos casos de impedimentos físicos (39,3\% se ayudaban con aparatos ortopédicos). Así que como ya se mencionó anteriormente, el estado de salud bucal del adulto mayor, aunque esté influido por el proceso de envejecimiento, dependerá de otras condiciones como los hábitos de higiene oral, grado de incapacidad física, la valoración que se tenga en relación a la salud bucodental, el acceso a servicios de salud, entre otros (19).

Respecto al edentulismo, en este estudio el $100 \%$ de los adultos mayores había perdido dientes, donde el $48,5 \%$ eran totalmente edéntulos $(48,5 \%$ eran hombres y $52,1 \%$ mujeres) y el $51,5 \%$ era par- 
cialmente edéntulos, con un promedio de 4,7 dientes, teniendo más dientes en boca los hombres ( 5 dientes) que las mujeres (3,7 dientes). Por tanto, se observa en esta población una cantidad muy reducida de dientes en boca, indicando que es una población sin funcionalidad adecuada, lo cual también ha sido observado en otros estudios, como el de Joya y Quintero (promedio de dientes: 11) (19) y el de Díaz et al (con una frecuencia alta de personas con menos de 19 dientes) (14). En el ENSAB IV, encontraron en población de adulto mayor evaluada un porcentaje de edentulismo total de 32,9 y un promedio de dientes perdidos en edéntulos parciales de 20 (12).

Respecto a la aparatología en boca, el $47 \%$ de los adultos mayores de este estudio, presentaban prótesis, predominando la prótesis total removible (38,2\%). Además, se encontró un alto porcentaje de adultos mayores $(77,9 \%)$ que requerían prótesis o cambio de estas. Esto indicaba una vez más, que una buena parte de los adultos mayores valorados en este estudio presentaban una condición desfavorable en la funcionalidad bucal. Las consecuencias biológicas por la mala funcionalidad bucal, repercuten en aspectos como la alimentación y nutrición, y en la estética para las relaciones sociales, afectando por tanto la calidad de vida (12)(22). Respecto a otros estudios, Dias Da Silva et al evidenciaron necesidad de prótesis total superior en el 48,5\% de la población y de prótesis total inferior en el $45,5 \%$ (24). Bermúdez et al. en población institucionalizada detectaron una necesidad protésica del 30\% para el maxilar superior y 44,7\% para el inferior (21) y en el estudio de Joya y Quintero fue más alta que los otros estudios, con unas necesidades protésicas del $60,5 \%$ (19).

En estudios realizados en adultos mayores, es frecuente encontrar lesiones de tejidos blandos en boca. En este estudio el número de lesiones fue bajo comparado con otros estudios (19)(21). El 19\% de la población presentaba alguna lesión, donde predominaban las venas varicosas $(8,8 \%)$, presentes en mujeres $(10 \%)$ y en hombres (8\%), y lengua saburral (6\%) y aftas menores (4\%) presentes únicamente en hombres. Las venas varicosas se asocian con la edad debido a la pérdida de tejido conectivo que soporta los vasos (17) y la lengua saburral, se asocia con afecciones sistémicas tales como problemas cardiacos, gástricos y alergias (18).

Aunque la calidad de vida según el estado bucodental puede llegar a ser subjetiva, se pueden utilizar instrumentos que permitan evaluarla. En este estudio se utilizó el índice GOHAI, el cual mostró que la mayoría de la población (76,5\%) tenía una autopercepción baja de la salud oral, tanto en hombres como en mujeres. Al igual que en el estudio de Esquivel y Jiménez, quienes utilizaron el mismo índice GOHAI, no se observaron diferencias en la autopercepción entre el género masculino y femenino. Los autores encontraron, una asociación entre las alteraciones clínicas como fue la condición de los dientes (COP-D), con la puntuación del instrumento (22). Otros estudios han demostrado también la asociación de la salud bucodental con la calidad de vida en la población de los adultos mayores y cómo repercuten en las actividades cotidianas (28)(29). 
$\mathrm{Al}$ analizar las dimensiones se encontró que la función psicosocial fue el componente reportado con resultados negativos con mayor frecuencia, en especial el ítem relacionado con las veces que el adulto mayor se ha sentido contento sobre la forma como se ven sus dientes cuando se mira al espejo (35.3\%) y en segundo lugar, la preocupación que presentan los adultos mayores porque sus dientes o dentadura estén bien $32.4 \%$. Con respecto a la función física, el $32.4 \%$ manifestó incomodidad al comer. $Y$ con respeto a la sensibilidad, solamente el 19,1\% reportó haber tenido molestias en sus dientes "algunas veces" a causa del frío, el calor o las cosas dulces; el 14.7 \% reportó haber sentido dichas molestias "siempre" y el 2.9\% "frecuentemente". Resultados similares a estos obtuvieron Díaz et al (14) en el que frente a la función psicosocial el $44 \%$ de los adultos mayores manifestó incomodidad al comer frente a otras personas por problemas con sus dientes, el 63.3\% de los pacientes evaluados manifestaron incomodidad al comer, y el $42.5 \%$ manifestaron haber tenido molestias frente al consumo de alimentos fríos o calientes. Las funciones físicas y de dolor coincidieron en los dos estudios como las más reportadas, sin embargo, en la función psicosocial las preocupaciones de los adultos mayores fueron diferentes para cada estudio.

La puntuación GOHAI promedio del total de la muestra fue $43.45,( \pm 8,2)$ lo que corresponde a la categoría de malo. Al analizar la distribución de la muestra, se encontró que la mayoría se encontraba en este nivel ( 53 pacientes, equivalentes al 77.94\%). Resultados similares obtuvieron

1644 Gallardo et al (30) en un estudio realizado en Perú en 164 adultos mayores en el que la puntuación promedio del total de la muestra fue 41,17 , ( $\pm 10,2)$ lo que corresponde a la categoría de malo. Al analizar la distribución de la muestra, se encontró que la mayoría se encontraba en este nivel (127 pacientes, equivalentes al 77.4\%).

\section{Conclusiones}

En este estudio se encontró en los adultos mayores examinados, altos índices de caries, enfermedad periodontal, edentulismo y baja valoración en la autopercepción de la salud oral geriátrica con respecto a otros estudios. El número de dientes en boca, así como el porcentaje en la necesidad de prótesis nuevas, indicaron que los adultos mayores no tenían una funcionalidad adecuada. Además, se detectó en los índices de placa dental, gingival y periodontal la necesidad de desarrollar una estrategia dirigida a ellos y a los cuidadores que promueva y mejore la higiene oral, así como tratamientos odontológicos específicos que contribuyan a mejorar las condiciones adecuadas para su bienestar. Esto conlleva a que más que aceptar unas condiciones de enfermedad características de la edad, se puedan establecer medidas, políticas y programas públicos que sean apropiados para disminuir estos índices.

\section{Agradecimientos}

A los directores y a los responsables de los adultos mayores de los Centros de Protección Social San Camilo, Divino niño y San José, por facilitar el desarrollo de la investigación. Y a todos aquellos que de una u otra forma colaboraron en el levantamiento y análisis de datos para llevar a término la investigación. 


\section{Referencias}

1. National Institute on Aging. Humanity's Aging [Internet]. Estados Unidos: $\mathrm{NIH}$; 2015 [citado 15 de marzo 2016]. Disponible en: https://www.nia.nih.gov/research/ publication/global-health-and-aging/humanitys-aging

2. Departamento Administrativo Nacional de Estadística. Estimaciones 1985-2005 y proyecciones de población 2005-2020 [Internet]. Colombia: República de Colombia. 2011. [citado 10 de marzo 2016] Colombia; Disponible en: https://www.dane. gov.co/index.php/estadisticas-por-tema/demografia-y-poblacion/proyecciones-de-poblacion

3. World Health Organization. Proposed working definition of an older person in Africa for the MDS Project. [Internet]. Suiza: WHO. 2002. [citado 15 de diciembre 2015] Disponible en: http://www. who.int/healthinfo/survey/ageingdefnolder/en/

4. Organización Mundial de la Salud. Informe mundial sobre el envejecimiento y la salud [Internet]. Estados Unidos; 2015. [citado 15 de diciembre 2015]. Disponible en: apps.who.int/iris/bitstre am/10665/186466/1/9789240694873 spa.pdf

5. Hernandez de Ramos M. Rehabilitación oral para el paciente geriátrico [Internet]. Primera ed. Bogotá: Universidad Nacional de Colombia; 2001. 193 p. p. [citado 10 de marzo 2016]. Disponible en: https://books.google.com.co/ books? id=bEBIRPqO_SAC\&pg=PA15\&Ipg=PA15\&dq=HERN\%C3\%81NDEZ,+Marilia.+Rehabilitaci\% C3\%B3n+Oral+Para+el+Paciente+Geri\%C3\%A1trico.+Universidad+Nacional+de\&sour$\mathrm{ce}=\mathrm{bl} \&$ ots $=$ Yf00kQbcXe\&sig=WowvqguYXsTIPbQwrQyzX5DS7Cw\&hl=es\&ei=mIFTSt6hBs2

6. Colussi CF, De Freitas SF CM. The prosthetic need $\mathrm{WHO}$ index: a comparison between self-perception and professional assessment in an elderly population. Gerodontology. 2009;26(3):187-92.

7. Abrams AP. Physiology of Aging of Older A dults Systemic and Oral Health Considerations. Dent Clin NA [Internet]. Elsevier Inc; 2014;58(4):729-38. Disponible en: http://dx.doi.org/10.1016/j. cden.2014.06.002

8. Li X, Koltveit KM, Tronstad L, Olsen I. Systemic diseases caused by oral infection. Clinical Microbiology Reviews. 2000. 13(4): 547-558.

9. Meurman JH, Sanz M. Oral health, atherosclerosis, and cardiovascular disease. Crit Rev Oral Biol Med [Internet]. 2004;15(6):403-13. [citado 10 de marzo 2016]. Disponible en: http://dx.doi. org/10.1016/B978-1-4557-5134-1.00081-0

10. Kim J, Amar S. Peridontal disease and systemic conditions: a bidirectional relationship. Odontology. 2006. 94(1):10-21.

11. Scannapieco F. Pneumonia in nonambulatory patients. The role of oral bacteria and oral hygiene. J Am Dent Assoc. 2008;139(3):251-6.

12. República de Colombia. Ministerio de salud. IV Estudio Nacional de Salud Bucal ENSAB - IV 2013-2014. 2015;378p.

13. Ministerio de Salud y protección Social Colombia. Alcances de la ley 1276 de 2009 [Internet]. Colombia: Ministerio de Salud; 2009. [citado 02 de diciembre 2015] Disponible en: https://www.minsalud.gov.co/Documentos y Publicaciones/ Alcances de la Ley 1276 de 2009.pdf

14. Díaz S, Arrieta K, Ramos K. Impacto de la Salud Oral en la Calidad de Vida de Adultos Mayores. 2012;5(1):9-16.

15. Albala C, Sanchez H, Cea X, Fuentes A, Marin R. Self-assessed oral-health sta- 
tus and quality of life of older Chilean $\S$. 2013;56:513-7.

16. Sáez R, Dra Cl, Carmona M, Dra II, Jiménez Z, lii $Q$. Cambios bucales en el adulto mayor. Rev Cubana Estomatol. 2007. [citado 02 de diciembre 2015] 44(4): 1-14. Disponible en: http://scielo. sld.cu/scielo.php?script=sci_arttext\&pi$d=$ S0034-75072007000400011

17. García JC, Enrique P, Otero T, Silva AM, Iglesias MS, Lázaro $C$, et al. Estado de salud bucal de la población geriátrica perteneciente a la Clínica Ormani Arenado. Rev Ciencias médicas. 2011;15(2):7686.

18. Meneses G. EJ. Salud bucodental y calidad de vida oral en los mayores. [Internet]. España: Universidad Complutense de Madrid; 2010. [citado 15 diciembre de 2015]. Tesis. 182p. Disponible en: http:// eprints.ucm.es/12303/1/T32521.pd

19. Joya LD, Quintero LE. Caracterización de la salud bucal de las personas mayores de 65 años de la ciudad de Manizales, 2008. Hacia promoc salud. 2015;20(1):140-52.

20. Schaefer Ferreira AL, Reibnitz C, Lorenzini Erdmann A. El cuidado a la salud bucal del anciano: buenas prácticas fundamentadas en el paradigma de la promoción de la salud. Gerokomos. 2009;20(2):65-72.

21. Bermúdez, W. F., Concha, S.; Camargo DM. Perfil orofacial de las personas mayores institucionalizadas de la ciudad de Bucaramanga y su área metropolitana. USTASALUD. 2003;1:13-9.

22. Esquivel, R.I; Jiménez J. Necesidades de atención odontológica en adultos mayores mediante la aplicación del GOHAI. Rev la Asoc Dent Mex. 2010;67(3):127-32.
23. Alves da Silva, R.H; Martins Castro, RF; Soares Cunha, DC; Thais Almeida, C; De Magalhães Bastos, J.R; Camargo LMA. Cárie dentária em população ribeirinha do Estado de Rondônia, Região Amazônica, Brasil, 2005-2006. Cad Saúde Pública. 2008;24(10):2347-53.

24. Wada RS. Saúde bucal em adultos e idosos na cidade de Rio Claro, São Paulo , Brasil Oral health in adults and the elderly in Rio Claro, São Paulo, Brazil. Oral Health. 2004;20(2):626-31.

25. Taboada A., O.; Cortés C. X. HP. R. Perfil de salud bucodental en un grupo de adultos mayores del estado de Hidalgo. Rev ADM. 2014;71(2):77-82.

26. Mariño, R.; Giacam RA. Factors related to unmet oral health needs in older adults living. 2014;58:454-9.

27. Mariño R. La salud bucodental de los ancianos: realidad, mitos y posibilidades. Boletín la Of Sanit Panam. 1994;116(5):419-26.

28. Sánchez-garcía S, C M, Juárez-Cedillo T, Reyes-morales H, Fuente-Hernández $\mathrm{J}$ De. Estado de la dentición y sus efectos en la capacidad de los ancianos para desempeñar sus actividades habituales. Salud Publica Mex. 2007;49(3):173-81.

29. Slade GD, Spencer AJ, Locker D, Hunt RJ, Strauss RP, Beck JD. Variations in the social impact of oral conditions among older adults in South Australia, Ontario, and North Carolina. J Dent Res. 1996;75(7):1439-50.

30. Gallardo A, Picasso MA, Huillca N, Ávalos JC. Calidad de vida relacionada con la salud oral en adultos mayores de una población peruana. KIRU. 2013;10(2):145-150. 